Reports From the Field

\title{
A Service Evaluation of Acute Neurological Patients Managed on Clinically Inappropriate Wards
}

\author{
Richard J. Holmes, MSc, and Sophie Stratford, BSc
}

Objective: Despite the benefits of early and frequent input from a neurologist, there is wide variation in the availability of this service, especially in district general hospitals, with many patients managed on clinically inappropriate wards. The purpose of this service evaluation was to explore the impact this had on patient care.

Methods: A retrospective service evaluation was undertaken at a National Health Service hospital by reviewing patient records over a 6-month period. Data related to demographics, processes within the patient's care, and secondary complications were recorded. Findings were compared with those of stroke patients managed on a specialist stroke ward.
Results: A total of 63 patients were identified, with a mean age of 72 years. The mean length of stay was 25.9 days, with a readmission rate of $16.7 \%$. Only $15.9 \%$ of patients were reviewed by a neurologist. There was a high rate of secondary complications, with a number of patients experiencing falls $(11.1 \%)$, pressure ulcers $(14.3 \%)$, and health care-acquired infections $(33.3 \%)$ during their admission.

Conclusions: The lack of specialist input from a neurologist and the management of patients on clinically inappropriate wards may have negatively impacted length of stay, readmission rates, and the frequency of secondary complications.

Keywords: evaluation; clinical safety; neurology; patientcentered care; clinical outcomes; length of stay. $t$ is estimated that $10 \%$ of acute admissions to district general hospitals (DGHs) of the National Health Service (NHS) in the United Kingdom are due to a neurological problem other than stroke.1 In 2011, a joint report from the Royal College of Physicians and the Association of British Neurologists (ABN) recommended that all of these patients should be admitted under the care of a neurologist and be regularly reviewed by a neurologist during their admission. ${ }^{2}$ The rationale for this recommendation is clear. The involvement of a neurologist has been shown to improve accuracy of the diagnosis ${ }^{3}$ and significantly reduce length of stay. ${ }^{4,5}$ Studies have also shown that the involvement of a neurologist has led to a change in the management plan in as high as $79 \%{ }^{6}$ to $89 \%{ }^{3}$ of cases, suggesting that a high proportion of neurological patients not seen by a neurologist are being managed suboptimally.

Despite this, a recent ABN survey of acute neurology services found ongoing wide variations in the availability of this specialist care, with a large proportion of DGHs having limited or no access to a neurologist and very few having dedicated neurology beds. ' While it is recognized that services have been structured in response to the reduced numbers of neurologists within the United Kingdom, ${ }^{8}$ it is prudent to assess the impact that such services have on patient care.

With this in mind, we planned to evaluate the current provision of care provided to neurological patients in a real-world setting. This was conducted in the context of a neurology liaison service at a DGH with no dedicated neurology beds.

\section{Methods}

A retrospective service evaluation was undertaken at a DGH in the southeast of England. The NHS hospital has neurologists on site who provide diagnostic and thera-

From Western Sussex Hospitals NHS Foundation Trust, Physiotherapy Department, Chichester, UK (Richard J. Holmes), and Western Sussex Hospitals NHS Foundation Trust, Department of Occupational Therapy, Chichester, UK (Sophie Stratford). 
peutic consultations on the wards, but there are no dedicated beds for patients with neurological conditions. Patients requiring neurosurgical input are referred to a tertiary neurosciences center.

Patients were selected from the neurotherapy database if they were referred into the service between August 1, 2019, and January 31, 2020. The neurotherapy database was used as this was the only source that held thorough data on this patient group and allowed for the identification of patients who were not referred into the neurologist's service. Patients were included if they had a new neurological condition as their primary diagnosis or if they had an exacerbation of an already established neurological condition. If a patient was admitted with more than 1 neurological diagnosis then the primary diagnosis for the admission was to be used in the analysis, though this did not occur during this evaluation. Patients with a primary diagnosis of a stroke were included if they were not managed on the acute stroke ward. Those managed on the stroke ward were excluded so that an analysis of patients managed on wards that were deemed clinically inappropriate could be undertaken. Patients were not included if they had a preexisting neurological condition (ie, dementia, multiple sclerosis) but were admitted due to a non-neurological cause such as a fall or infection. All patients who met the criteria were included.

A team member independently reviewed each set of patient notes. Demographic data extracted from the medical notes included the patient's age (on admission), gender, and diagnosis. Medical, nursing, and therapy notes were reviewed to identify secondary complications that arose during the patient's admission. The secondary complications reviewed were falls (defined as the patient unexpectedly coming to the ground or other lower level), health care-acquired infections (HAls) (defined as any infection acquired during the hospital admission), and pressure ulcers (defined as injuries to the skin or underlying tissue during the hospital admission). Other details, obtained from the patient administration system, included the length of stay (days), the number of ward moves the patient experienced, the speciality of the consultant responsible for the patient's care, the discharge destination, and whether the patient was readmitted for any cause within 30 days.
All data collected were stored on a password-protected computer and no patient-identifiable data were included.

The results were collated using descriptive statistics. The $\chi^{2}$ test was used to compare categorical data between those patients who were and were not reviewed by a neurologist, and the Mann-Whitney $U$ test was used to compare differences in the length of stay between these 2 groups.

No national data relating to this specific patient group were available within the literature. Therefore, to provide a comparator of neurological patients within the same hospital, data were collected on stroke patients managed on the stroke ward. This group was deemed most appropriate for comparison as they present with similar neurological symptoms but are cared for on a specialist ward. During the evaluation period, 284 stroke patients were admitted to the stroke ward. A sample of 75 patients was randomly selected using a random number generator, and the procedure for data collection was repeated. It was not appropriate to make direct comparative analysis on these 2 groups due to the inherent differences, but it was felt important to provide context with regards to what usual care was like on a specialist ward within the same hospital.

Ethical approval was not required as this was a service evaluation of routinely collected data within a single hospital site.

\section{Results}

In total, 63 patients were identified: 26 females and 37 males. The median age of patients was 74 years (range, 39-92 years). These demographic details and comparisons to stroke patients managed on a specialist ward can be seen in Table 1. To quantify the range of diagnoses, the condition groups defined by GIRFT Neurology Methodology ${ }^{9}$ were used. The most common diagnoses were tumors of the nervous system (25.4\%) and traumatic brain and spine injury (23.8\%). The other conditions included in the analysis can be seen in Table 2.

Despite having a neurological condition as their primary diagnosis, only $15.9 \%$ of patients were reviewed by a neurologist during their hospital admission. Patients were most commonly under the care of a geriatrician $(60.3 \%)$, but they were also managed by orthopedics (12.6\%), acute medicine (7.9\%), respiratory (6.3\%), cardiol- 
Table 1. Demographic and Outcome Data for Comparison

\begin{tabular}{|c|c|c|}
\hline & $\begin{array}{l}\text { Neurological patients managed on } \\
\text { clinically inappropriate wards }(\mathrm{N}=63)\end{array}$ & $\begin{array}{l}\text { Stroke patients managed on the } \\
\text { specialist stroke ward }(N=75)\end{array}$ \\
\hline \multicolumn{3}{|l|}{ Age, y } \\
\hline Median & 74 & 76 \\
\hline Range & $39-92$ & $26-98$ \\
\hline \multicolumn{3}{|l|}{ Sex, n (\%) } \\
\hline Male & $37(58.7)$ & $31(41.3)$ \\
\hline Female & $26(41.3)$ & $44(58.7)$ \\
\hline \multicolumn{3}{|l|}{ Length of stay, days } \\
\hline Mean & 25.9 & 11.4 \\
\hline Range & $2-78$ & $1-81$ \\
\hline \multicolumn{3}{|c|}{ Frequency of secondary complications, $n(\%)$} \\
\hline Falls & $7(11.1)$ & $8(10.7)$ \\
\hline Pressure ulcers & $9(14.3)$ & $1(1.3)$ \\
\hline Health care-acquired infections & $21(33.3)$ & $8(10.7)$ \\
\hline \multicolumn{3}{|l|}{ Discharge destination, $\mathrm{n}(\%)$} \\
\hline Usual residence & $33(52.4)$ & $62(82.7)$ \\
\hline Rehabilitation & $10(15.9)$ & $4(5.3)$ \\
\hline Nursing home & $9(14.3)$ & $5(6.7)$ \\
\hline Residential home & $4(6.3)$ & $0(0.0)$ \\
\hline Tertiary center & $3(4.8)$ & $2(2.7)$ \\
\hline Hospice & $1(1.6)$ & $0(0.0)$ \\
\hline Died & $3(4.8)$ & $2(2.7)$ \\
\hline $\begin{array}{l}\text { Readmission rate of those alive on } \\
\text { discharge, } n(\%)\end{array}$ & $10(16.7)$ & $8(11.0)$ \\
\hline
\end{tabular}

ogy (4.8\%), gastroenterology (3.2\%), and surgery (3.2\%). One patient (1.6\%) was managed by intensivists.

The average length of stay was 25.9 days (range, 2-78 days). This was more than double the average length of stay on the stroke ward (11.4 days) (Table 1) and the national average for patients with neurological conditions (9.78 days). ${ }^{10}$ During their stay, $33 \%$ had 2 or more ward moves, with 1 patient moving wards a total of 6 times. Just over half (52.4\%) of the patients returned to their usual residence on discharge. The remainder were discharged to rehabilitation units (15.9\%), nursing homes (14.3\%), residential homes (6.3\%), tertiary centers (4.8\%), and hospice (1.6\%). Unfortunately, 3 patients (4.8\%) passed away. Of those still alive ( $n=60$ ), $16.7 \%$ were readmitted to the hospital within 30 days, compared to a readmission rate of $11 \%$ on the stroke ward. None of the patients who were readmitted were seen by a neurologist during their initial admission.

The frequency of secondary complications was reviewed as a measure of the multidisciplinary management of this patient group. It was noted that $11.1 \%$ had a fall on the ward, which was similar to a rate of $10.7 \%$ on the stroke ward. More striking was the fact that $14.3 \%$ of patients developed a pressure ulcer and 33.3\% developed an HAl during their admission, compared with rates of $1.3 \%$ and $10.7 \%$, respectively, on the stroke ward (Table 1).

There were no significant differences found in length of stay between those who were and were not reviewed by a neurologist $(P=.73)$. This was also true for categorical data, whereby readmission rate $(P=.13)$, frequency of 
Table 2. Frequency of Neurological Diagnoses

\begin{tabular}{lc}
\hline Diagnosis & $\begin{array}{c}\text { No. of Patients } \\
\text { (\%) (N = 63) }\end{array}$ \\
\hline Tumors of the nervous system & $16(25.4)$ \\
\hline Traumatic brain and spine injury & $15(23.8)$ \\
\hline Subarachnoid hemorrhage and stroke & $9(14.3)$ \\
\hline Surgical spine/peripheral nerve & $5(7.9)$ \\
\hline Central nervous system infections & $5(7.9)$ \\
\hline Peripheral nerve disorders & $4(6.3)$ \\
\hline Multiple sclerosis and inflammatory disorders & $3(4.8)$ \\
\hline Rare and other neurological disorders & $2(3.2)$ \\
\hline Functional disorders & $1(1.6)$ \\
\hline Headaches and migraine & $1(1.6)$ \\
\hline Motor neuron disease and spinal muscular & $1(1.6)$ \\
\hline atrophy & $1(1.6)$ \\
\hline $\begin{array}{l}\text { Parkinsonism and other extrapyramidal } \\
\text { disorders/Tic disorder }\end{array}$
\end{tabular}

falls $(P=.22)$, frequency of pressure ulcers $(P=.67)$, and HAls $(P=.81)$ all failed to show a significant difference between groups.

\section{Discussion}

The findings of this service evaluation show markedly poorer outcomes for neurological patients compared to stroke patients managed on a specialist stroke ward. It is suggested that these results are in part due to the lack of specialist input from a neurologist in the majority of cases and the fact that all were managed on clinically inappropriate wards. Only $15.9 \%$ of neurological patients were seen by a neurologist. This is a slight improvement compared to previous studies in DGHs that showed rates of $10 \%{ }^{1}$ and $11 \%,{ }^{11}$ but it is still a far cry from the goal of $100 \%$ set out in recommendations. ${ }^{2}$ In addition, the increased readmission rate may be suggestive of suboptimal management, especially given that none of those readmitted had been reviewed by a neurologist. There are undoubtedly other factors that may influence readmissions, such as comorbidities, the severity/complexity of the condition, and the strength of community services. However, the impact of a lack of input from a specialist should not be underestimated, and further evaluation of this factor (with confounding factors controlled) would be beneficial.
The result of an extended length of stay was also a predictable outcome based on previous evidence., ${ }^{4,5}$ With the potential for suboptimal management plans and inaccurate diagnoses, it is inevitable that the patient's movement through the hospital system will be impeded. In our example, it is possible that the extended length of stay was influenced by the fact that patients included in the evaluation were managed on nonspecialist wards and a large proportion had multiple ward changes.

Given that the evidence clearly shows that stroke patients are most effectively managed by a multidisciplinary team (MDT) with specialist skills, ${ }^{12}$ it is likely that other neurological patients, who have similar multifactorial needs, would also benefit. The patients in our evaluation were cared for by nursing staff who lacked specific skills and experience in neurology. The allied health professionals involved were specialists in neurotherapy but were not based on the ward and not directly linked to the ward MDT. A review by Epstein found that the benefits of having a MDT, in any speciality, working together on a ward included improved communication, reduced adverse events, and a reduced length of stay. ${ }^{13}$ This lack of an effective MDT approach may provide some explanation as to why the average length of stay and the rates of some secondary complications were at such elevated levels.

A systematic review exploring the impact of patients admitted to clinically inappropriate wards in a range of specialities found that these patients were associated with worse outcomes. ${ }^{14}$ This is supported by our findings, in which a higher rate of pressure ulcers and HAls were observed when compared to rates in the specialist stroke ward. Again, a potential explanation for this is the impact of patients being managed by clinicians who lack the specialist knowledge of the patient group and the risks they face. Another explanation could be due to the high number of ward moves the patients experienced. Blay et al found that ward moves increased length of stay and carried an associated clinical risk, with the odds of falls and HAls increasing with each move..$^{15} \mathrm{~A}$ case example of this is apparent within our analysis in that the patient who experienced 6 ward moves not only had the longest length of stay (78 days), but also developed a pressure ulcer and 2 HAls during their admission. 
This service evaluation had a number of limitations that should be considered when interpreting the results. First, despite including all patients who met the criteria within the stipulated time frame, the sample size was relatively small, making it difficult to identify consistent patterns of behavior within the data.

Furthermore, caution should be applied when interpreting the comparators used, as the patient groups are not equivalent. The use of comparison against a standard is not a prerequisite in a service evaluation of this nature, but comparators were included to help frame the context for the reader. As such, they should only be used in this way rather than to make any firm conclusions.

Finally, as the evaluation was limited to the use of routinely collected data, there are several variables, other than those reported, which may have influenced the results. For example, it was not possible to ascertain certain demographic details, such as body mass index and socioeconomic factors, nor lifestyle factors such as smoking status, alcohol consumption, and exercise levels, all of which could impact negatively on the outcomes of interest. Furthermore, data were not collected on follow-up services after discharge to evaluate whether these had any impact on readmission rates.

\section{Conclusion}

This service evaluation highlights the potential impact of managing neurological patients on clinically inappropriate wards with limited input from a neurologist. There is the potential to ameliorate these impacts by cohorting these patients in neurologist-led beds with a specialist MDT. While there are limitations in the design of our study, including the lack of a controlled comparison, the small sample size, and the fact that this is an evaluation of a single service, the negative impacts to patients are concerning and warrant further investigation.

Corresponding author: Richard J. Holmes, MSc, Physiotherapy Department, St. Richard's Hospital, Chichester, West Sussex, PO19 6SE; richard.holmes8@nhs.net.
Financial disclosures: None.

doi: 10.12788/jcom.0049

\section{References}

1. Kanagaratnam M, Boodhoo A, MacDonald BK, Nitkunan A. Prevalence of acute neurology: a 2-week snapshot in a district general hospital. Clin Med (Lond). 2020;20(2):169-173.

2. Royal College of Physicians. Local adult neurology services for the next decade. Report of a working party. June 2011. Accessed October 29, 2020. https://www.mstrust.org.uk/sites/default/files/ files/Local\%20adult\%20neurology\%20services\%20for\%20the\%20 next\%20decade.pdf

3. McColgan P, Carr AS, McCarron MO. The value of a liaison neurology service in a district general hospital. Postgrad Med J. 2011;87(1025):166-169.

4. Forbes R, Craig J, Callender M, Patterson V. Liaison neurology for acute medical admissions. Clin Med (Lond). 2004;4(3):290.

5. Craig J, Chua R, Russell C, et al. A cohort study of early neurological consultation by telemedicine on the care of neurological inpatients. J Neurol Neurosurg Psychiatry. 2004;75(7): 1031-1035.

6. Ali E, Chaila E, Hutchinson M, Tubridy N. The 'hidden work' of a hospital neurologist: 1000 consults later. Eur J Neurol. 2010; 17(4):e28-e32.

7. Association of British Neurologists. Acute Neurology services survey 2017. Accessed October 29, 2020. https://cdn.ymaws.com/ www.theabn.org/resource/collection/219B4A48-4D25-472697AA-OEB6090769BE/ABN_2017_Acute_Neurology_Survey.pdf

8. Nitkunan A, Lawrence J, Reilly MM. Neurology Workforce Survey. January 28, 2020. Accessed October 28, 2020. https://cdn. ymaws.com/www.theabn.org/resource/collection/219B4A484D25-4726-97AA-0EB6090769BE/2020_ABN_Neurology_ Workforce_Survey_2018-19_28_Jan_2020.pdf

9. Fuller G, Connolly M, Mummery C, Williams A. GIRT Neurology Methodology and Initial Summary of Regional Data. September 2019. Accessed October 26, 2020. https://gettingitrightfirsttime.co.uk/wp-content/uploads/2017/07/GIRFT-neurologymethodology-090919-FINAL.pdf

10. The Neurological Alliance. Neuro Numbers 2019. Accessed October 28, 2020. https://www.neural.org.uk/wp-content/ uploads/2019/07/neuro-numbers-2019.pdf

11. Cai A, Brex P. A survey of acute neurology at a general hospital in the UK. Clin Med (Lond). 2010;10(6):642-643.

12. Langhorne P, Ramachandra S; Stroke Unit Trialists' Collaboration. Organised inpatient (stroke unit) care for stroke: network meta-analysis. Cochrane Database Syst Rev. 2020;4(4):CD000197.

13. Epstein NE. Multidisciplinary in-hospital teams improve patient outcomes: A review. Surg Neurol Int. 2014;5(Suppl 7):S295-S303.

14. La Regina M, Guarneri F, Romano E, et al. What Quality and Safety of Care for Patients Admitted to Clinically Inappropriate Wards: a Systematic Review. J Gen Intern Med. 2019;34(7): 1314-1321.

15. Blay $N$, Roche M, Duffield $C, X u X$. Intrahospital transfers and adverse patient outcomes: An analysis of administrative health data. J Clin Nurs. 2017;26(23-24):4927-4935. 